\title{
Short time-scale heating of the Earth's mantle by ice-sheet dynamics
}

\author{
Ladislav Hanyk ${ }^{1}$, Ctirad Matyska ${ }^{1}$, and David A. Yuen ${ }^{2}$ \\ ${ }^{1}$ Charles University, Faculty of Mathematics and Physics, Department of Geophysics, 18000 Prague, Czech Republic \\ ${ }^{2}$ Department of Geology and Geophysics and Minnesota Supercomputing Institute, University of Minnesota, Minneapolis MN 55455, U.S.A.
}

(Received December 2, 2004; Revised May 28, 2005; Accepted June 6, 2005)

\begin{abstract}
We have studied the possibility of short time-scale energy transfer from the ice sheet loading and unloading processes to the Earth's interior via viscous dissipation associated with the transient viscoelastic flow in the mantle. We have focussed on the magnitude of glacially induced deformations and the corresponding shear heating for an ice sheet of the spatial extent of Laurentide region in Maxwellian viscoelastic compressible models with a Newtonian viscosity. We have used a discretization method based on the method of lines for integrating the time-dependent evolutionary equations of self-gravitational, viscoelastic flow. We have found that shear heating from the transient viscoelastic flow can represent a non-negligible mantle energy source with cryogenic origins. Volumetric heating by viscous deformation associated with these flows can be locally greater than chondritic heating by radioactivity. In the presence of an abrupt change in the ice loading history, the time average of the integral of the dissipation over depth corresponds to a mantle heat flow of the order of magnitude of $\mathrm{mW} / \mathrm{m}^{2}$ below the periphery of ancient ice sheets or below their central areas. However, the peak values of this integral in time are almost two orders higher. Our results would suggest that some degree of volcanism may be associated with dramatic episodes in ice loading.
\end{abstract}

Key words: Viscoelasticity, heat dissipation, postglacial rebound.

\section{Introduction}

The Earth is a nonlinear dynamical system with a fluid atmosphere and oceans, varying ice distribution and a mantle which is convecting vigorously with mobile plates and rebounding from the ice sheet loading of the last ice age. Most of the work related to postglacial rebound, up to now, has been concerned with the geophysical signatures associated with the surface deformation, such as the uplift rates (e.g., Peltier, 1974, 2003). As postglacial rebound has traditionally been studied by modeling the linear response of a self-gravitating viscoelastic planet, the gravity field anomalies have been also used as the standard output from the modeling (e.g., Wu and Peltier, 1982; Mitrovica and Peltier, 1989). Although these surficial signatures depend on the deformation field of the entire planet and not only on the surface deformation, not much attention has been paid to the interior transient flow induced by the ice-sheet forcing.

Glacial loading operates on a much faster timescale than mantle convection (Siddall et al., 2003; North Greenland Ice Core Project members, 2004). One may suspect then that a fast mode of energy transfer may couple these two processes. We will focus here on an energy transfer mechanism between the atmosphere, the cryosphere and the Earth's interior. This mechanism arises from the effects from internal deformation, in particular that of viscous dissipation from cryogenic forcing, in order to test the idea that changes of ice-sheet loading may contribute in a nonnegligible way to localized internal heating in the Earth's

Copyright (C) The Society of Geomagnetism and Earth, Planetary and Space Sciences (SGEPSS); The Seismological Society of Japan; The Volcanological Society of Japan; The Geodetic Society of Japan; The Japanese Society for Planetary Sciences; TERRAPUB mantle, as has been shown already in certain dynamical situations of mantle convection (Balachandar et al., 1995) and lithospheric dynamics (Regenauer-Lieb and Yuen, 1998). Nakada and Yokose (1992) have already recognized that there is a cause-and-effect relationship between ice loading and subsequent volcanism. The influence of transient viscoelastic flow from tidal forcing in causing vulcanism of the Jovian moons has long been recognized (Ross and Schubert, 1987; Segatz et al., 1988). As the crucial parameter influencing the deformation of the Earth as well as the internal dissipation is the mantle viscosity stratification, which is still poorly constrained, especially under the lithosphere, we will perform a parameter study varying viscosity stratification to obtain potential limits of dissipative heating. In order to reveal the fundamental physics of this novel thermal-mechanical phenomenon, we will also study different time- and spatial- variations of the ice loading.

First, we will present the model along with a numerical technique, based on the method of lines (e.g., Schiesser, 1994), for integrating the governing partial differential equations of viscoelastic mantle flow. Then we will illustrate the physics of the flow and the attendant shear heating by means of advanced visualization techniques. This is followed by several examples with different viscosity profiles. Finally we discuss the implications of this novel heating mechanism for planetary dynamics.

\section{Formulation of Time-dependent Integration of Viscoelastic Equations}

We consider the partial differential equations (PDEs) governing the spatial-temporal responses of spherical selfgravitating viscoelastic Maxwellian Earth models (e.g., 
Peltier, 1974; Hanyk et al., 1996),

$$
\begin{aligned}
& \frac{\partial}{\partial t}\left[\nabla \cdot \boldsymbol{\tau}^{E}-\varrho_{0} \nabla \varphi_{1}+\nabla \cdot\left(\varrho_{0} \mathbf{u}\right) \nabla \varphi_{0}-\nabla\left(\varrho_{0} \mathbf{u} \cdot \nabla \varphi_{0}\right)\right] \\
& \quad=\nabla \cdot\left[\frac{\mu}{\eta}(\boldsymbol{\tau}-K \nabla \cdot \mathbf{u} \boldsymbol{I})\right] \\
& \boldsymbol{\tau}^{E}=\lambda \nabla \cdot \mathbf{u} \boldsymbol{I}+\mu\left[\nabla \mathbf{u}+(\nabla \mathbf{u})^{T}\right] \\
& \nabla \cdot\left(\nabla \varphi_{1}+4 \pi G \varrho_{0} \mathbf{u}\right)=0
\end{aligned}
$$

where $\boldsymbol{I}, \boldsymbol{\tau}$ and $\boldsymbol{\tau}^{E}$ are the identity tensor, the stress tensor and the elastic part of $\boldsymbol{\tau}$, respectively, $\mathbf{u}$ is the displacement vector, $\varphi_{0}$ and $\varphi_{1}$ the initial and incremental gravitational potentials, $\varrho_{0}$ the density, $K$ the bulk modulus, $\lambda$ and $\mu$ are Lame's elastic moduli, $\eta$ the dynamic viscosity and $G$ the gravitational constant.

By employing the spherical harmonic decomposition and considering axisymmetric surface loading, these equations can be converted into a set of the linear first-order PDEs with respect to time $t$ and the radius $r$,

$$
\begin{aligned}
& \frac{\partial^{2} \boldsymbol{y}_{n}(t, r)}{\partial t \partial r}-\boldsymbol{A}_{n}(r) \frac{\partial \boldsymbol{y}_{n}(t, r)}{\partial t} \\
& \quad=\frac{\mu(r)}{\eta(r)}\left[\boldsymbol{D}_{n}(r) \frac{\partial \boldsymbol{y}_{n}}{\partial r}(t, r)+\boldsymbol{E}_{n}(r) \boldsymbol{y}_{n}(t, r)\right],
\end{aligned}
$$

where $\boldsymbol{y}_{n}$ is a 6-component solution vector describing the two components of the spheroidal displacement, two stress components and perturbation of the gravitational potential as well as the gravitational accceleration for the spherical harmonic degree $n$. The $6 \times 6$ matrices $\boldsymbol{A}_{n}(r), \boldsymbol{D}_{n}(r)$ and $\boldsymbol{E}_{n}(r)$ are dependent on $\varrho_{0}(r), \mu(r), K(r)$ and the gravitational acceleration. Equation (3) and the matrices in the full $8 \times 8$ form for both spheroidal and toroidal components are derived in Hanyk et al. (2002). The cut-off degree of the spherical harmonic decomposition has been taken at least 360 in all following calculations.

We employ a semi-discretization scheme of (3) commonly known as the method of lines (e.g., Schiesser, 1994) over the spatial grid $\left\{r_{i}\right\}$ spanning over the Earth mantle, i.e., $r_{0}=b<r_{i}<r_{N}=a$ with $a$ and $b$ being the radii of the Earth and the $\mathrm{CMB}$, respectively. This results in a differential-algebraic system, which takes a form of ordinary differential equations in time and allows us to integrate in time (along the "time lines" in each point of the spatial grid in parallel) with differential integrators suitable for numerically stiff problems, such as postglacial rebound (Hanyk, 1999).

In calculating viscous dissipation, we are not interested in the volumetric deformations as they are purely elastic in our models and no heat is thus dissipated during volumetric changes, as would be the case in phase changes. Therefore we have focussed only on the shear deformations. The Maxwellian constitutive relation (Peltier, 1974) rearranged for the shear deformations takes the form of

$$
\frac{\partial \boldsymbol{\tau}_{S}}{\partial t}=2 \mu \frac{\partial \boldsymbol{e}_{S}}{\partial t}-\frac{\mu}{\eta} \boldsymbol{\tau}_{S}
$$

where

$$
\boldsymbol{\tau}_{S}=\boldsymbol{\tau}-K \nabla \cdot \boldsymbol{u I}, \quad \boldsymbol{e}_{S}=\boldsymbol{e}-\frac{1}{3} \nabla \cdot \boldsymbol{u} \boldsymbol{I},
$$

$\boldsymbol{e}$ is the deformation tensor. This equation can be rewritten as the sum of elastic and viscous contributions to the total deformation,

$$
\frac{\partial \boldsymbol{e}_{S}}{\partial t}=\frac{\partial \boldsymbol{\tau}_{S}}{2 \mu \partial t}+\frac{\boldsymbol{\tau}_{S}}{2 \eta} \equiv \frac{\partial \boldsymbol{e}_{S}^{e l}}{\partial t}+\frac{\partial \boldsymbol{e}_{S}^{v i s}}{\partial t} .
$$

The rate of mechanical energy dissipation $\phi$ for a viscoelastic body (cf. Joseph, 1990, p. 50) is then given by

$$
\phi(t, \boldsymbol{r}) \equiv \boldsymbol{\tau}_{S}: \frac{\partial \boldsymbol{e}_{S}^{v i s}}{\partial t}=\frac{\boldsymbol{\tau}_{S}: \boldsymbol{\tau}_{S}}{2 \eta} .
$$

Note that the traction acting at horizontal planes only is obtained by integrating (3); it is therefore necessary to integrate also the constitutive relation (4) to be able to evaluate (7). We have calculated $\phi$ a posteriori using the method of lines from the results derived from the momentum and constitutive equations. For timescales shorter than $10^{5}$ years, we may neglect the temperature equation and the attendant thermal-mechanical feedback for global geodynamics (e.g., Regenauer-Lieb and Yuen, 2003). A similar expression for evaluating a posteriori the amount of seismic dissipation in the elastic normal-mode formalism can be found in Gilbert and Buland (1977).

\section{Results}

Most geophysicists usually associate the phenomenon of viscous heating with mantle convection (e.g., van den Berg and Yuen, 1997) or fault-zone dynamics (e.g., RegenauerLieb and Yuen, 1998, 2003). The idea of viscous heating within the context of postglacial rebound was first suggested by Buttles et al. (1998) and first quantified by Hanyk et al. (2003). The physics behind this process is illustrated in Fig. 1, where the streamlines due to the transient mantle flow induced by the loading and unloading of ice sheets are illustrated. Heating occurs over a timescale of the order of $10^{4}$ years, much shorter than those associated with mantle convection. Viscous heating is concentrated in the proximity of the ice sheet and can extend deep into the mantle, depending on the viscosity stratification and the horizontal extent of the ice sheets and the timescale of the forcing.

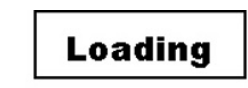
Unloading

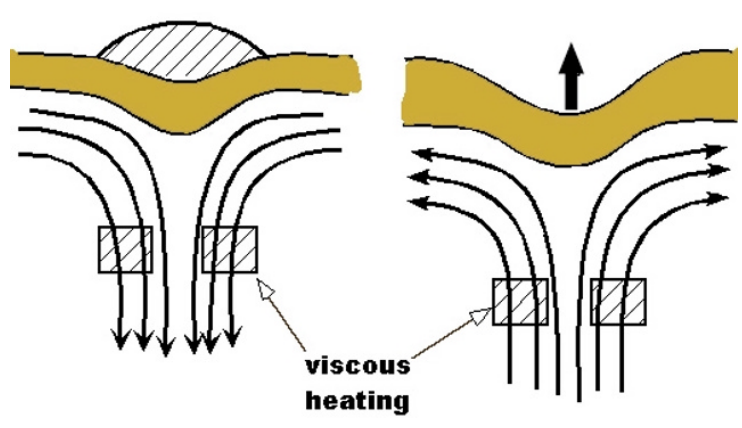

Fig. 1. Schematic diagram showing the viscous dissipation produced in the ice-loading process. Transient flows, shown as streamlines, develop in response to the time-dependent surface loading and unloading conditions 
L1

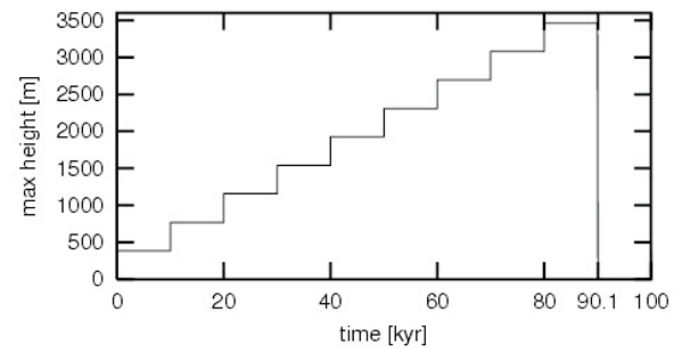

L2
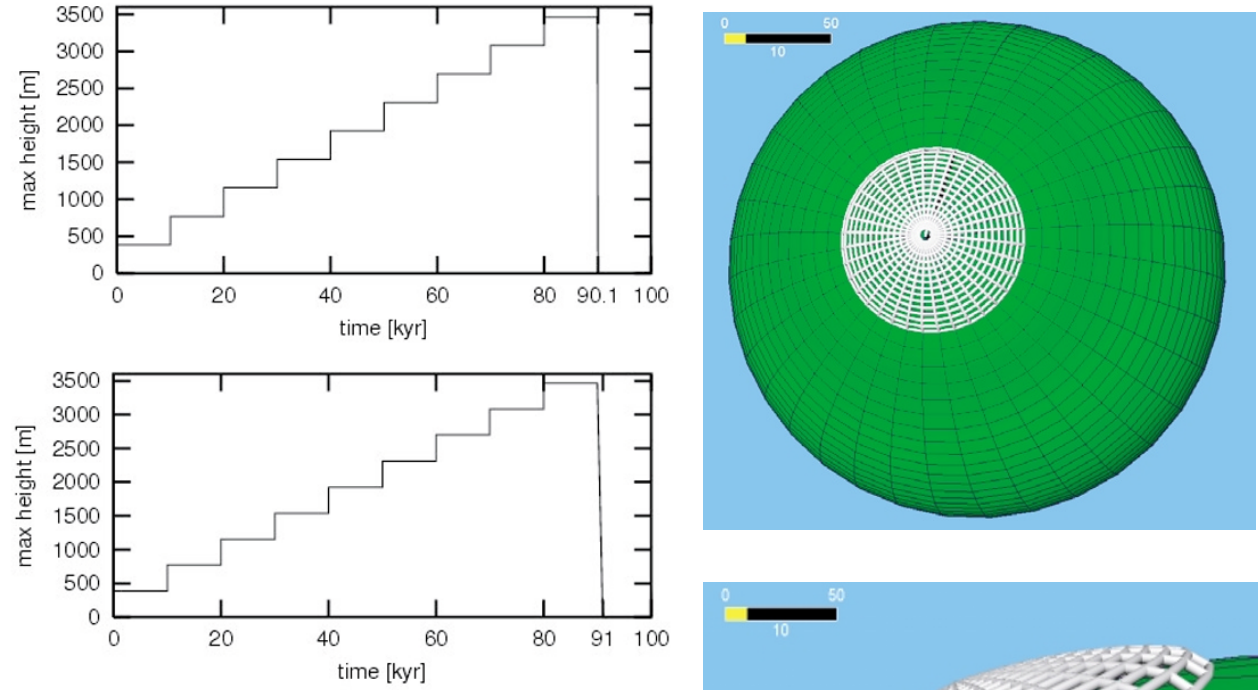

L3
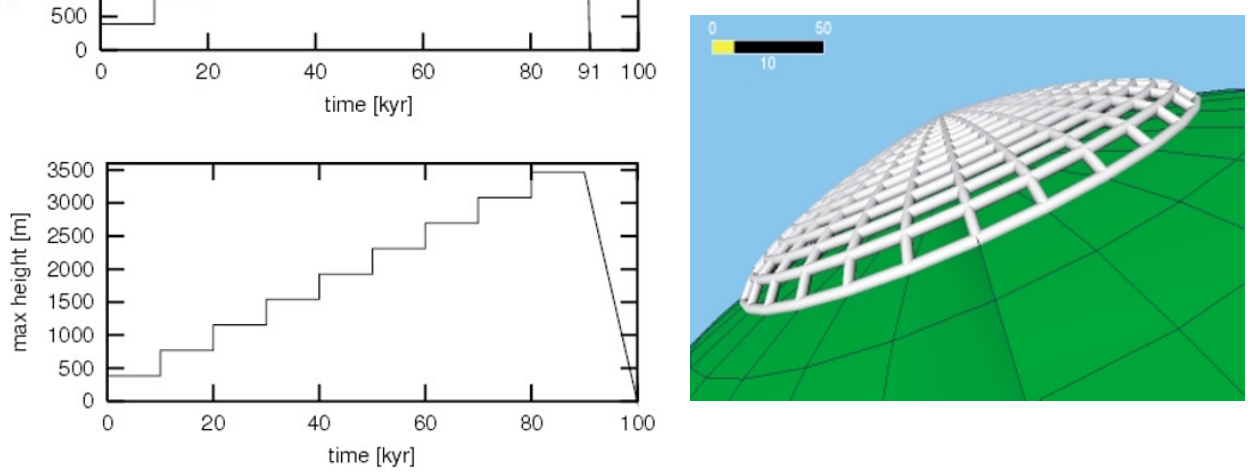

Fig. 2. L1, L2 and L3 are time histories of the ice sheet with disintegration times of 100 years, 1 kyr and 10 kyr, respectively. The growing phase lasts for $90 \mathrm{kyr}$. The spatial distribution of the load corresponds to the parabolic-shaped ice sheet of the Laurentide size $\left(15^{\circ}\right.$ radius, $3500 \mathrm{~m}$ maximum height).

In Fig. 2 we show a surface ice sheet we have chosen in the numerical experiments. The model with cylindrical symmetry has the parabolic cross-section with vertical edges, the radius of $15^{\circ}$ and the ice maximal thickness of $3500 \mathrm{~m}$, which are the parameters corresponding to the Laurentide ice sheet. This spatial function is then multiplied by cyclic time-forcing functions of the period $100 \mathrm{kyr}$. In order to test potential influence of both abrupt and gradual changes during the evolution of the ice cover we have considered three types of time-forcing functions. They are characterized by an increase from 0 to 1 during the first $90 \mathrm{kyr}$ followed by a linear decrease to 0 during the last $10 \mathrm{kyr}$. The loading phase is a step-wise function made of the same nine ascending steps, the unloading phase of the three ice histories are linear with the duration of 100 years, $1 \mathrm{kyr}$ or $10 \mathrm{kyr}$, respectively. The choice of these step functions was motivated by the fact that high-latitude climate can exhibit large and abrupt fluctuations with timescales of around a few centuries (Clark et al., 2001; Yuan et al., 2004). As we have employed the initial-value approach, we started the computations from the static equilibrium and performed several cycles to suppress the influence of transient dynamical effects.

We have employed the Maxwell Newtonian viscoelastic rheology, which has been succesful in explaining sea-level changes and gravity anomalies induced by glacial loading (e.g., Wu and Peltier, 1982). The elastic part of the rheology is given by the PREM model (Dziewonski and An- derson, 1981). As the style of viscosity stratification is still poorly constrained, we have used three different $1-D$ viscosity profiles for capturing the basic physics associated with the different viscosity stratifications (plotted in the left panels of Figs. 4-6). The first model M1 of viscosity is simply an isoviscous mantle with the viscosity of $10^{21} \mathrm{~Pa}$ and the elastic lithosphere of the thickness of $110 \mathrm{~km}$. This model can thus be considered to be the reference case. Since dynamic geoid modeling as well as rotational dynamics require at least an order of magnitude increase of viscosity in the lower mantle (Mitrovica and Forte, 2004), we have increased the lower-mantle viscosity in model $\mathrm{M} 2$ by adding a 'lower-mantle viscosity hill' exceeding the value $10^{23} \mathrm{~Pa} \mathrm{~s}$ (see also Hanyk et al., 1995), whereas viscosity in the upper mantle remains the same and the thickness of the elastic lithosphere is again $110 \mathrm{~km}$. Finally, the model M3 contains a low-viscosity zone (LVZ) just below the lithosphere, with $\eta=10^{19} \mathrm{Pas}$ and the thickness of $110 \mathrm{~km}$, which is compensated by an increased viscosity of the magnitude $2 \times 10^{22} \mathrm{~Pa}$ s in the transition zone in the depth interval 400 $670 \mathrm{~km}$. The remaining parts of the mantle have the same viscosity profile as in the second model.

We start with visualization of the vertical (Fig. 3(a)) and horizontal (Fig. 3(b)) displacements of the reference Earth model M1 undergoing one cycle of the ice sheet history L3 with the linear load disintegration lasting $10 \mathrm{kyr}$. We consider both the incompressible and compressible Earth models, i.e., models with the bulk modulus $K$ infinite or 
(a)
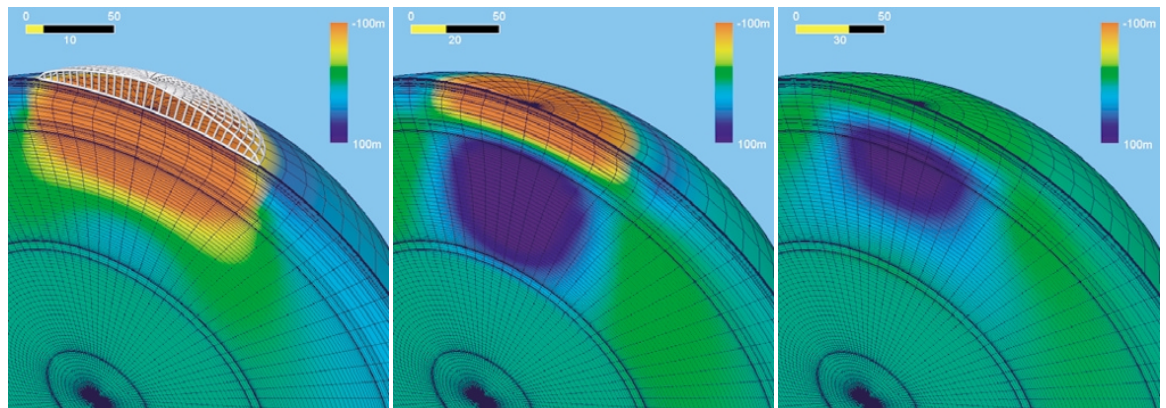

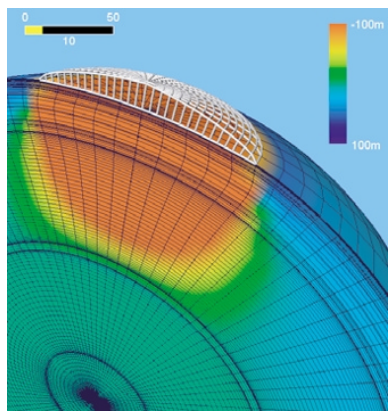

90 kyr

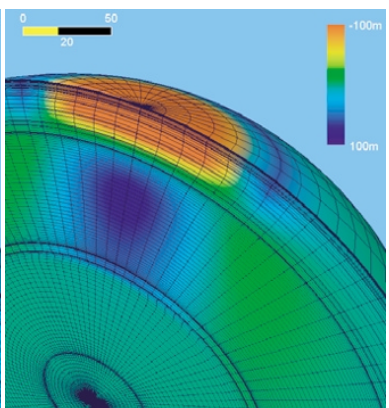

$100 \mathrm{kyr}$

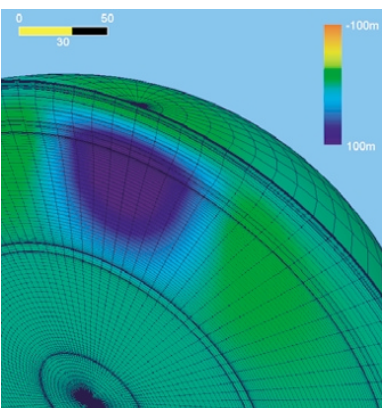

$150 \mathrm{kyr}$

(b)
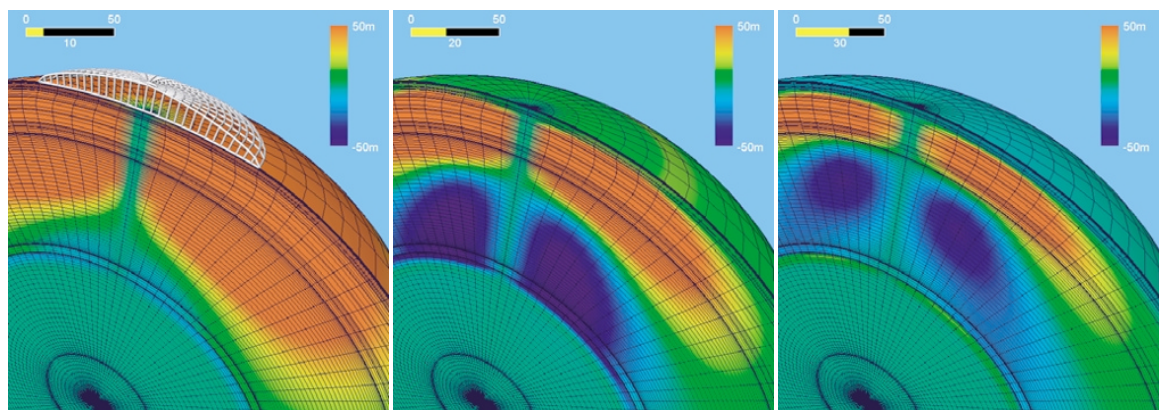

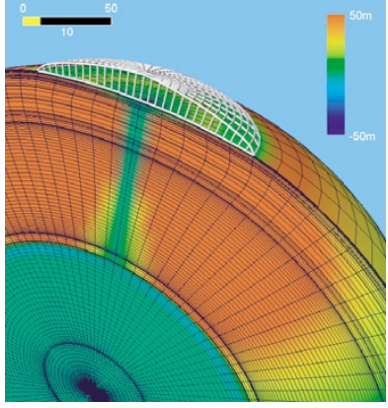

$90 \mathrm{kyr}$

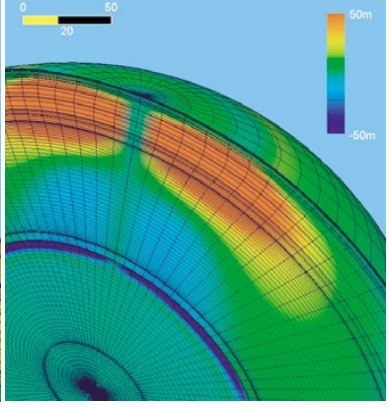

$100 \mathrm{kyr}$

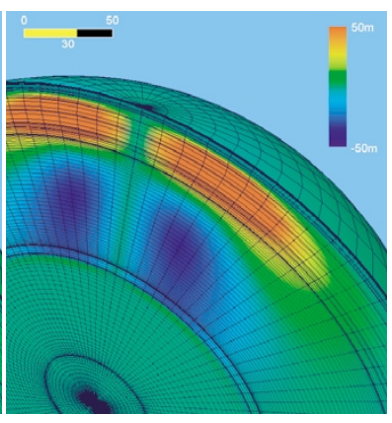

$150 \mathrm{kyr}$

Fig. 3. a) Vertical displacement in the mantle at the glacial maximum ( $90 \mathrm{kyr}$ ), after the unloading phase ( $100 \mathrm{kyr})$ and in the course of the relaxation process (150 kyr). The realistic Earth model (incompressible in the upper panels, compressible in lower panels) with parameters by PREM, the isoviscous mantle and the $110-\mathrm{km}$ elastic lithosphere is considered. Red color represents depressions, blue uplift areas; the distortions of the coordinate mesh are shown exaggerated. b) Corresponding horizontal displacement. Red color represents movements outward, blue indicates movements toward the center of the ice sheet.

taken from the PREM, to give the possibility to distinguish between the responses. The displacements are shown at the moments of the loading maximum $(90 \mathrm{kyr})$, at the ice sheet removal (100 kyr) and after next $50 \mathrm{kyr}$ of the freesurface relaxation process, upper panels for incompressible, lower for compressible models. The complicated, non- monotonic deformation patterns of realistic Earth models are demonstrated. One can easily see the faster depression inside the loaded compressible model. A remarkable effect is the opposite sign of surficial horizontal displacements of incompressible and compressible models in the loading maximum. Fig. 3, as well as the following three figures, has 


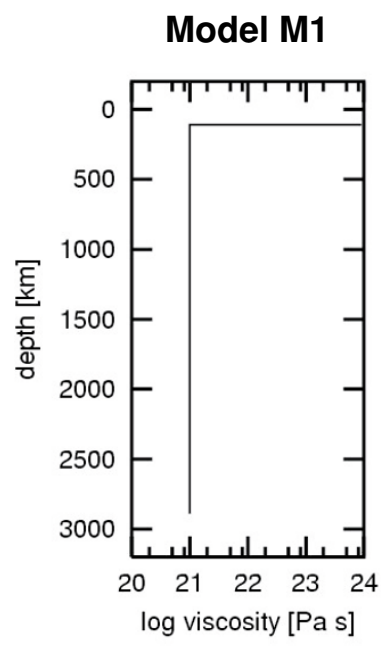

L1
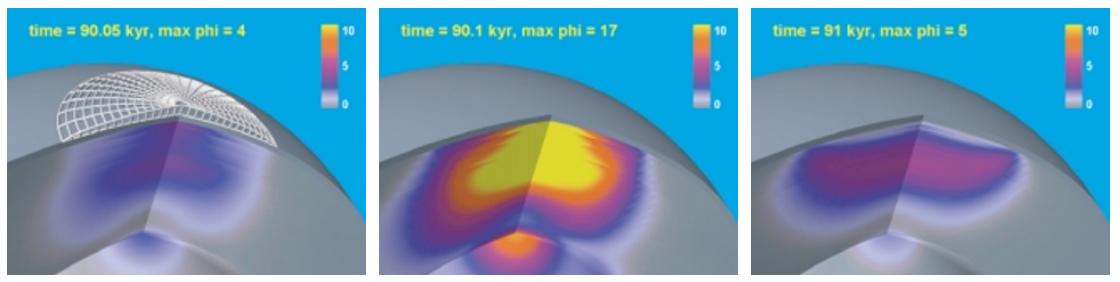

L2
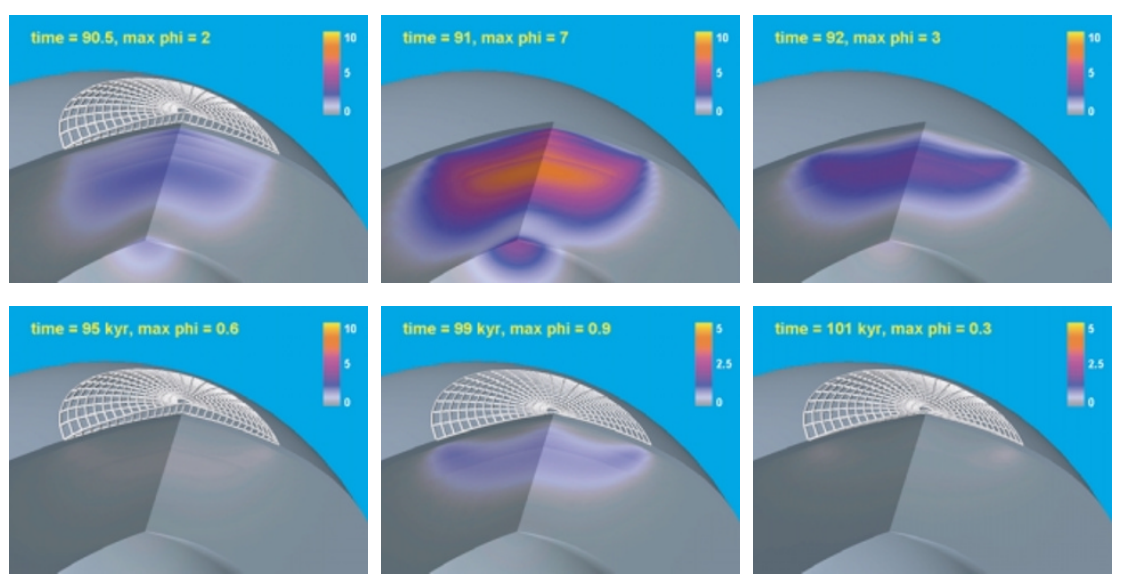

before

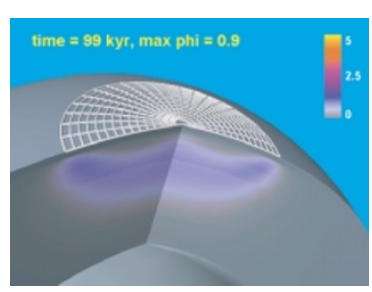

heating maximum

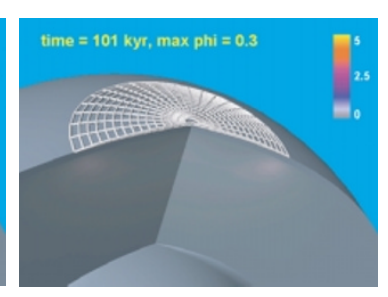

after

Fig. 4. Spatial distribution of dissipative heating corresponding to the isoviscous Earth model M1 and three loading histories L1 (fast), L2 (medium) and L3 (slow). Three successive snapshots are shown with the middle ones taken at the moments of heating maxima. Color scales are normalized with respect to the chondritic radiogenic heating of $3 \times 10^{-9} \mathrm{~W} / \mathrm{m}^{3}$.

been prepared by the advanced 3-D visualization software amira (http://www.amiravis.com, see also Erlebacher et al., 2001), which allows for easy interactivity.

Figure 4 shows the spatial distribution of the dissipative heating for the viscosity model M1 and for the ice histories L1, L2 and L3 (upper, middle and lower rows of each figure). The panels are the snapshots at the moments of ice sheet disintegration, when the dissipation $\phi$ reaches its maximum (middle column) and at the moments before (left column) and after (right colums); the actual time is written in each panel. The dissipative heating is scaled by the chondritic magnitude of radiogenic heating of $3 \times 10^{-9} \mathrm{~W} / \mathrm{m}^{3}$ (Leitch and Yuen, 1989), which is used for measuring viscous dissipation in mantle convection (Balachandar et al., 1995). As the differences in the results among the models are substantial, two color maps are used: in the upper two rows grey corresponds to zero and yellow to the chondritic value multiplied by 10 , whereas in the bottom row yellow yields the chondritic value multiplied by 5 . We emphasize, however, that local dissipation maxima can be much higher, as indicated by the yellow "max phi" subtitles in each panel. The dissipative heating for models M2 and M3 is visualized in Figs. 5 and 6, respectively.

We recognize clearly that both the distribution of dissipative heating and its amplitude are sensitive to several parameters. The most important factor for the amplitudes is the shape of the time history. Short time-scale changes in the ice history, as in L1, are able to produce higher dissipation rates than slower changes as in L2 and L3. While the local dissipation maximum for the isoviscous model M1 and the time function L3 with $10 \mathrm{kyr}$ unloading is approximately equal to the chondritic radiogenic heating, the dissipation maximum right after the abrupt $100 \mathrm{yr}$ unloading in L1 can reach the multiple of 17. Almost the same ratio between L3 and L1 can also be found for the model M3 in Fig. 6.

The viscosity stratification is important for the shape of the heated areas. The parabolic load pumps most of the energy into the mantle under its central part, in the isoviscous model M1 down to the core-mantle boundary, in the model M2 with high-viscous lower mantle down to the $670 \mathrm{~km}$ boundary. The presence of the LVZ in the model M3 results in the additional local heating maximum underneath the load rim and the maximal heating is raised by more than one order in magnitude in comparison to the models M1 and M2.

The total heating obtained by volumetric integration of dissipation over the whole mantle during the warming maxima can reach 1 TW. Although the total heat loss of the Earth is about 44 TW (Stein, 1995), the ice sheet covers less than $2 \%$ of the Earth's surface in our model and thus 1 TW represents a non-negligible level of heating. However, such a high value can be reached only for a relatively short time interval, which is clearly visible In Fig. 7 from the time dependence of the maximal local heating for the whole $100-\mathrm{kyr}$ glacial cycle. This is the reason why timeaveraged global heating is about $0.03 \mathrm{TW}$.

To obtain a better appreciation of the magnitude of dissipative heating, we introduce the surface distribution of the dissipation

$$
q_{m}(t, \vartheta)=\int_{\mathrm{CMB}}^{a} \phi(t, r, \vartheta) r^{2} d r / a^{2},
$$

where $t$ is the time, $a$ the Earth's radius and $\vartheta$ the colatitude. We do not consider a dependence on the longitude as we have employed only axisymmetric loading. As $q_{m}$ can be formally considered as an 'equivalent mantle 


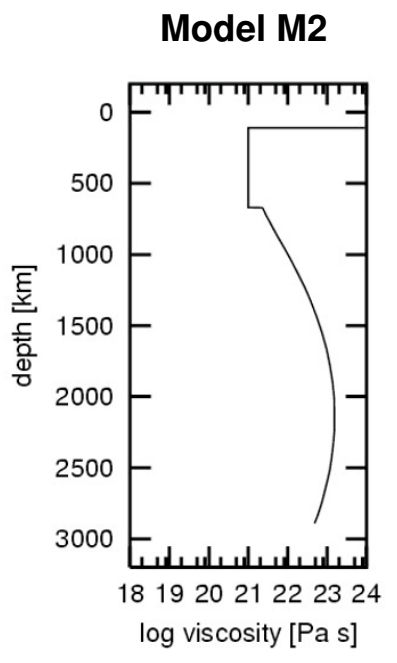

L1

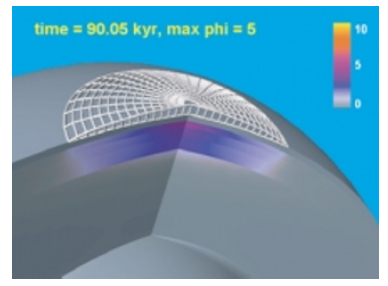

L2

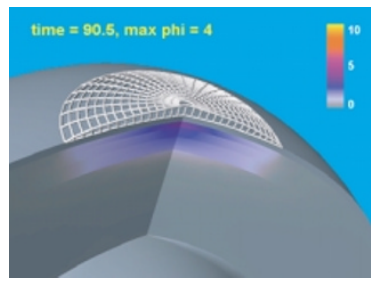

L3

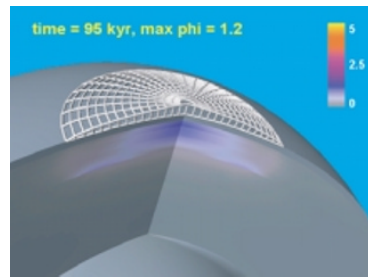

before
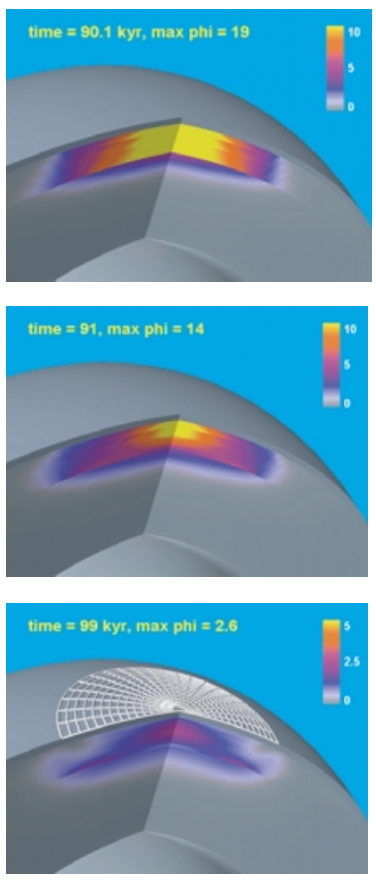

heating maximum
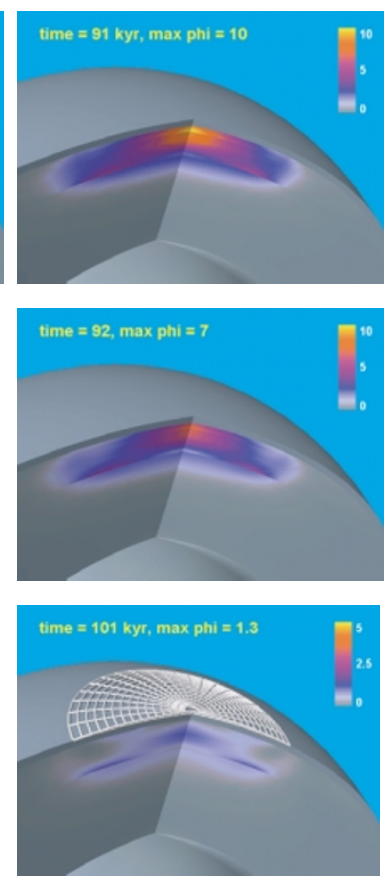

after

Fig. 5. Spatial distribution of dissipative heating in the Earth model M2 with high viscosity in the lower mantle.

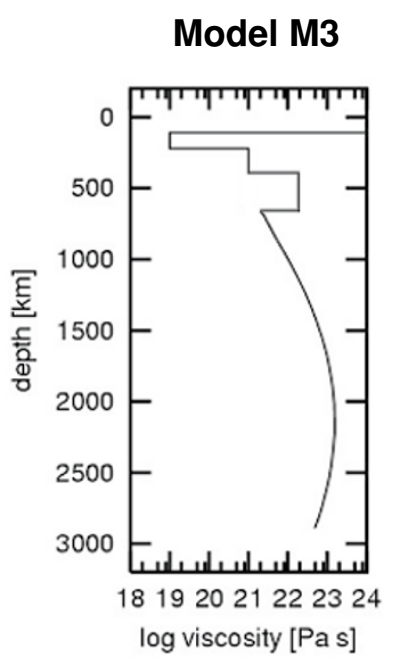

L1
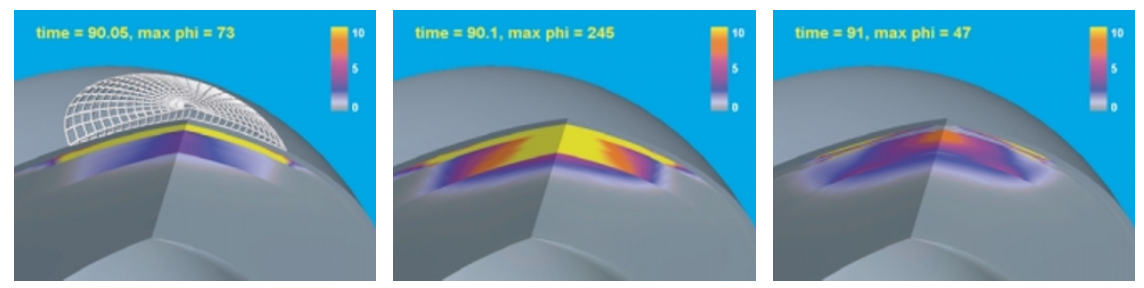

L2
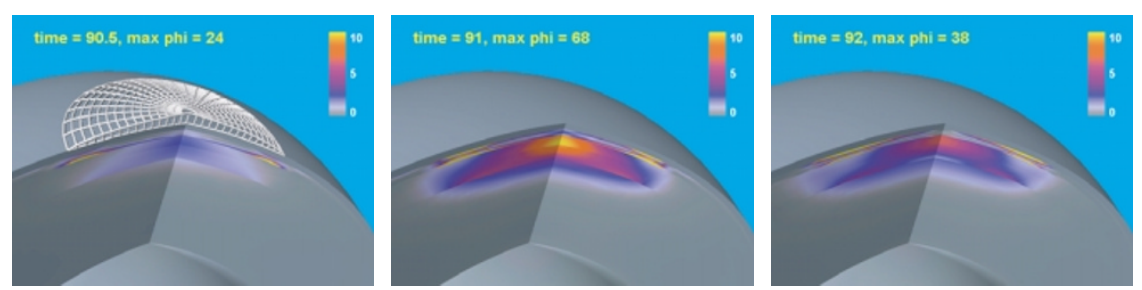

L3

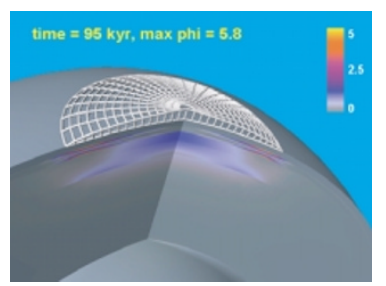

before heating maximum

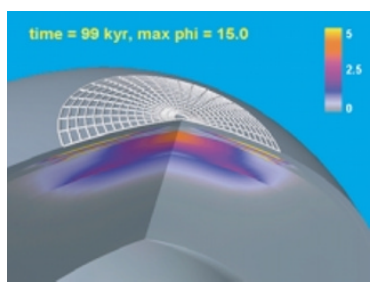

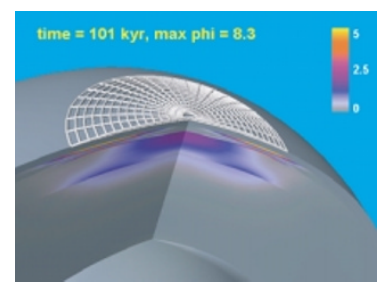

after

Fig. 6. Spatial distribution of dissipative heating in the Earth model M3 with high viscosity region in the mid-lower mantle and the low-viscosity zone underneath the elastic lithosphere.

heat flow' due to dissipation, we can compare its magnitude with estimates of realistic heat flow at the bottom of the lithosphere. For example, Pasquale et al. (1991) employed downward heat flow continuation method combined with estimates of crustal radiogenic heating and found typical mantle heat flow $19-25 \mathrm{~mW} / \mathrm{m}^{2}$ in the central part of Fennoscandia followed by an increase to $30-45 \mathrm{~mW} / \mathrm{m}^{2}$ un- der southern Sweden. This means that typical changes of mantle heat flow under the glacial shields can be of the order of $10 \mathrm{~mW} / \mathrm{m}^{2}$; this constraint may be a starting point for estimating the amount of viscous heating. We note that there is also an increased heat flow in Denmark and northern Germany (Čermák and Hurtig, 1979), however, heat flow values in the Canadian area are rather low (Jaupart et al., 

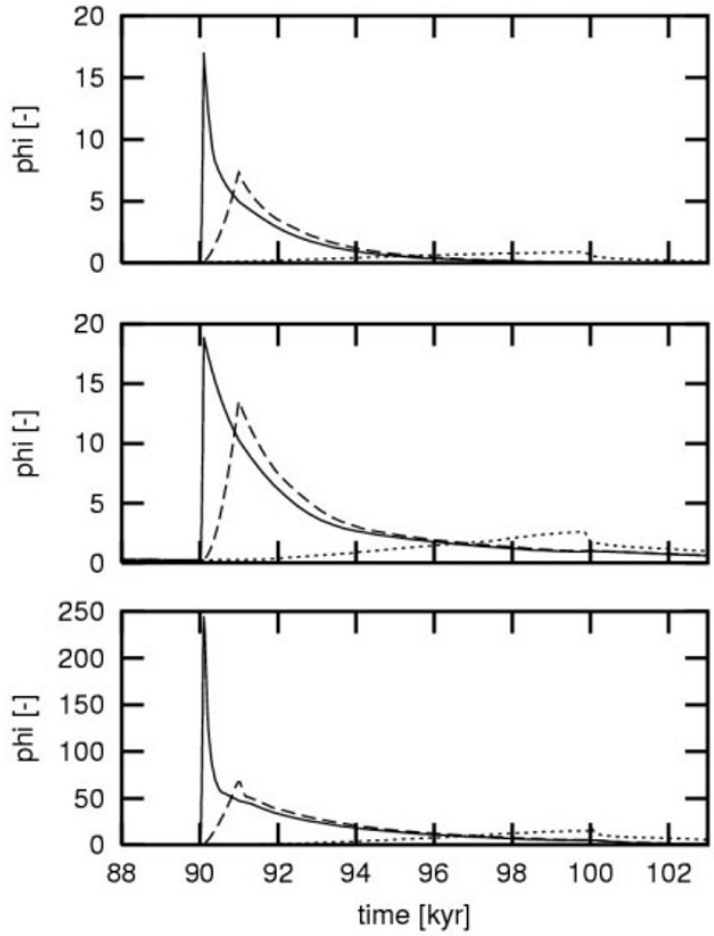

Fig. 7. Time evolution of normalized maximal local heating for a glacial cycle of duration of $100 \mathrm{kyr}$. The rows correspond to the three studied viscosity models M1, M2 and M3. The heating values for the time-spatial forcing L1, L2 and L3, as shown in Fig. 2, are represented by the solid, dashed and dotted curves in each panel.

1998).

We demonstrate in the left panels of Fig. 8 that $q_{m}$ can reach tens of $\mathrm{mW} / \mathrm{m}^{2}$ with absolute maximum of $80 \mathrm{~mW} / \mathrm{m}^{2}$. However, these are the maximal values and we should take into account that the characteristic thermal diffusion time of the lithosphere is much higher than typical time spans of maximal heating events. This is the reason why we should take into account an average of $q_{m}$ over the whole glacial cycle to be able to conclude whether the energy pumping into the mantle by ice sheets can influence the dynamics of the region below the moving ice sheets. We can see that the time-averaged values are of the order of $\mathrm{mW} / \mathrm{m}^{2}$, and the question arises whether it can be neglected in thermo-mechanical considerations of mantle dynamics, since over a period of $10^{5}$ years they can trigger secondary convective instabilities, especially with nonlinear viscous rheology.

From convection studies (Larsen et al., 1997, 1999) and also from shear-zone deformation modeling (RegenauerLieb and Yuen, 1998) there is much more viscous heating generated in a non-Newtonian rheology than in the Newtonian rheological model. It is well known that the velocity fields of non-Newtonian convection have much sharper spikes in time than Newtonian convection (Christensen, 1984; Malevsky and Yuen, 1992a). A factor of 100 increase in the viscous heating may be obtained since the velocity deformation rates need only be 10 times higher. The actual dissipative heating with nonlinear rheology can thus be much higher than the upper limits of this study based on the Newtonian viscosity. However, we have not studied
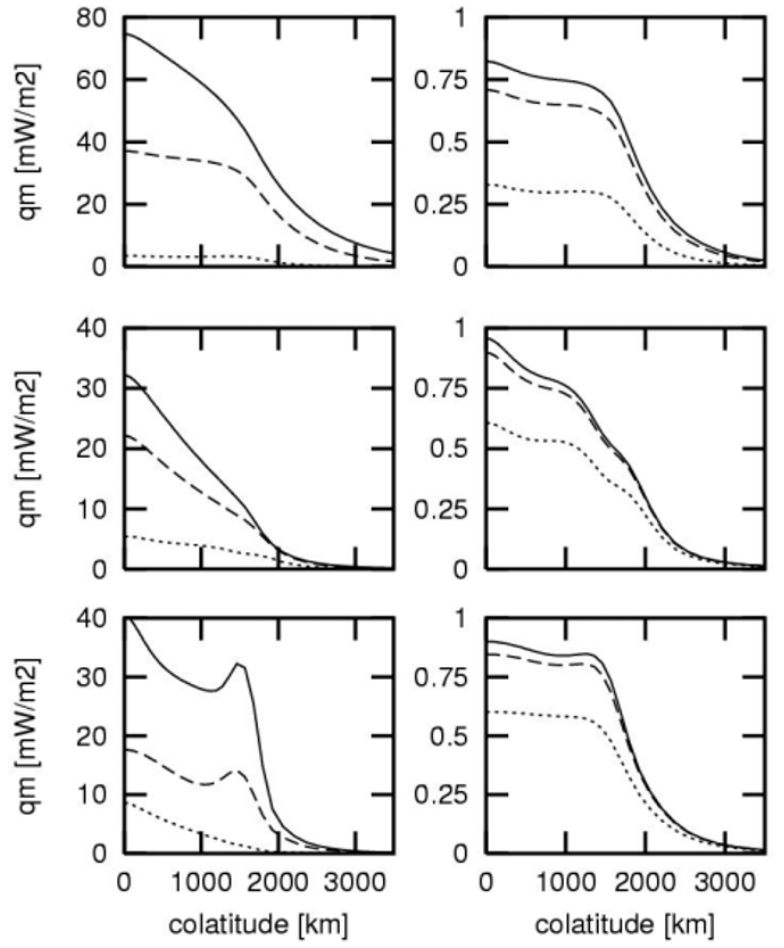

Fig. 8. Equivalent mantle heat flow $q_{m}\left(\mathrm{~mW} / \mathrm{m}^{2}\right)$ due to shear dissipation as a function of the distance from the load axis ('colatitude'). Left column: peak values taken at the end of unloading, right column: time-averaged values, solid, dashed and dotted curves: loading histories $\mathrm{L} 1, \mathrm{~L} 2$ and $\mathrm{L} 3$, respectively. The rows correspond to the viscosity modeles M1, M2 and M3. Heat flow generated by the parabolic disc increases generally toward the load centre, however, when the LVZ is considered, a local heat flow maximum can be found underneath the rim.

in detail the dynamical role played by the different elastic lithospheric thicknesses. From a physical point of view it is probable that there can be filtering out of short-wavelength surface deformation by the elastic lithosphere.

\section{Concluding Remarks}

The association of ice ages and volcanism has been mentioned in the literature (e.g., Nakada and Yokose, 1992; Goddéris et al., 2003). The effects of tidal forcing by Jupiter on causing melting in the Jovian moons are also well known (Peale et al., 1979; Ross and Schubert, 1987; Segatz et al., 1988). These two types of forcing are external to the planetary body in nature. In this work, we have explored, for the first time ever, the geophysical prospects of significant viscous dissipation being able to be generated over short geological timescale induced by surface ice-sheet forcing. For a family of viscosity profiles and different time-dependent forcing functions, we have demonstrated that the magnitude of shear heating caused by cryospheric forcing can be comparable to chondritic radioactive heating below the center of the ice load with the size of the Laurentide area, and much higher in a low-viscosity zone below the edges of the ice fields. The magnitude and the spatial distribution of shear heating is extremely sensitive to the choice of the time-forcing function because its abrupt changes result in the heating maxima. The presence of the low-viscosity zone enables focusing of thermal energy into this layer. This 
study will open the doors to future studies concerning the transfer of potential energy from the surface movements of ice sheets to the mantle.

In this work we have employed a linear Newtonian viscous rheology, whose coupling to thermal instabilities would give the least amount of finite-amplitude effect and the longest timescale in the response (Malevsky and Yuen, 1992b; Larsen and Yuen, 1997). Non-Newtonian viscous rheology and the inclusion of plasticity would enhance the thermal responses much more dramatically (RegenauerLieb and Yuen, 2004). Therefore the results presented here should be regarded as a lower bound in an assessment of its potential influence in a more realistic rheological setup.

Acknowledgments. We thank M. Běhounková and B. Kadlec for discussions, which have prompted us to look into viscous dissipation, and L. Yang for her drawing. Reviews by M. Nakada, M. Kameyama and B. Moore improved the manuscript significantly. The work has been supported by the Research Project DG MSM 113200004, by the Charles University Grant 238/2001/BGEO/MFF, by the Czech Grant Agency Projects 205/00/D113 and 205/03/0778 and by the CSEDI and ITR programs of the National Science Foundation.

\section{References}

Balachandar, S., D. A. Yuen, D. M. Reuteler, and G. Lauer, Viscous dissipation in three dimensional convection with temperature-dependent viscosity, Science, 267, 1150-1153, 1995.

Buttles, J., P. Malin, and A. Hunt, 2-D models of upper mantle viscosity variations due to ice sheet loading: Application to the Laurentide ice sheet, AGU Fall Meeting, abstract No. T32C-15, 1998.

Čermák, V. and E. Hurtig, Heat flow map of Europe, Enclosure for Terrestrial Heat Flow in Europe, edited by V. Čermák and L. Rybach, Springer, Berlin, New York, 1979.

Christensen, U., Convection with pressure-dependent and temperaturedependent non-Newtonian rheology, Geophys. J. R. Astr. Soc., 77, 343 384, 1984.

Clark, P. U., S. J. Marshall, G. K. C. Clarke, S. W. Hostetler, J. M. Licciardi, and J. T. Teller, Freshwater forcing of abrupt climate change during the last glaciation, Science, 293, 283-287, 2001.

Dziewonski, A. M. and D. L. Anderson, Preliminary reference Earth model, Phys. Earth Planet. Inter., 25, 297-356, 1981.

Erlebacher, G., D. A. Yuen, and F. Dubuffet, Current trends and demands in visualization in the geosciences, Electron. Geosciences, 4, http://link.springer-ny.com/link/service/journals/10069/technic/ erlebach/index.htm, 2001.

Gilbert, F. and R. Buland, Dissipation of energy radiated by earthquakes, Annali di Geofisica, 30, 471-489, 1977.

Goddéris, Y., Y. Donnadieu, A. Nédélec, B. Dupré, C. Dessert, A. Grard, G. Ramstein, and L. M. François, The Sturtian 'snowball' glaciation: fire and ice, Earth Planet. Sci. Lett., 211, 1-12, 2003.

Hanyk, L., Viscoelastic response of the Earth: Initial-value approach, Ph.D. Thesis, Charles University, Prague, Czech Republic, http: // geo.mff. cuni.cz/ ${ }^{\sim}$ lh/phd, 1999.

Hanyk, L., J. Moser, D. A. Yuen, and C. Matyska, Time-domain approach for the transient responses in stratified viscoelastic Earth models, Geophys. Res. Lett., 22, 1285-1288, 1995.

Hanyk, L., D. A. Yuen, and C. Matyska, Initial-value and modal approaches for transient viscoelastic responses with complex viscosity profiles, Geophys. J. Int., 127, 348-362, 1996.

Hanyk, L., C. Matyska, and D. A. Yuen, Determination of viscoelastic spectra by matrix eigenvalue analysis, in Ice Sheets, Sea Level and the Dynamic Earth, edited by J. X. Mitrovica and B. L. A. Vermeersen, pp. 257-273, AGU, 2002.

Hanyk, L., C. Matyska, D. A. Yuen, and B. J. Kadlec, Visualization of viscous heating in the Earth's mantle induced by glacial loading, Eos Trans. AGU, 84, Fall Meet. Suppl., Abstract NG11A-0166, 2003.
Jaupart, C., J. C. Mareschal, and L. Guillou-Frottier, Heat flow and thickness of the lithosphere in the Canadian Shield, J. Geophys. Res., 103, 15269-15286, 1998.

Joseph, D. D., Fluid Dynamics of Viscoelastic Liquids, Springer, New York etc., 1990.

Larsen, T. B. and D. A. Yuen, Fast plumeheads: Temperature-dependent versus non-Newtonian rheology, Geophys. Res. Lett., 24, 1995-1998, 1997.

Larsen, T. B., D. A. Yuen, J. L. Smedsmo, and A. V. Malevsky, Generation of fast timescale phenomena in thermo-mechanical processes, Phys. Earth Planet. Inter., 102, 213-222, 1997.

Larsen, T. B., D. A. Yuen, and M. Storey, Ultrafast mantle plumes and implications for flood basalt volcanism in the northern Atlantic region, Tectonophys., 311, 31-43, 1999.

Leitch, A. and D. A. Yuen, Internal heating and thermal constraints on the mantle, Geophys. Res. Lett., 16, 1407-1410, 1989.

Malevsky, A. V. and D. A Yuen, Large-scale numerical simulations of turbulent non-Newtonian thermal-convection using method of characteristics, Comput. Phys. Commun., 73, 61-71, 1992a.

Malevsky, A. V. and D. A. Yuen, Strongly chaotic non-Newtonian mantle convection, Geophys. Astro. Fluid Dyn., 65, 149-171, 1992b.

Mitrovica, J. X. and A. M. Forte, A new inference of mantle viscosity based upon joint inversion of convection and glacial isostatic adjustment data, Earth Planet. Sci. Lett., 225, 177-189, 2004.

Mitrovica, J. X. and W. R. Peltier, Pleistocene deglaciation and the global gravity field, J. Geophys. Res., 94, 13651-13671, 1989.

Nakada, M. and H. Yokose, Ice age as a trigger of active Quaternary volcanism and tectonism, Tectonophys., 212, 321-329, 1992.

North Greenland Ice Core Project members, High-resolution record of Northern Hemisphere climate extending into the last interglacial period, Nature, 431, 147-151, 2004.

Pasquale, V., M. Verdoya, and P. Chiozzi, Lithospheric thermal structure in the Baltic shield, Geophys. J. Int., 106, 611-620, 1991.

Peale, S. J., P. Cassen, and R. T. Reynolds, Melting of Io by tidal dissipation, Science, 203, 892-894, 1979.

Peltier, W. R., The impulse response of a Maxwell earth, Rev. Geophys. Space Phys., 12, 649-669, 1974.

Peltier, W. R., GRACE signatures of global glacial isostasy and modern polar ice sheet melting, Geophysical Research Abstracts, 5, 02943, 2003.

Regenauer-Lieb, K. and D. A. Yuen, Rapid conversion of elastic energy into plastic shear heating during incipient necking of the lithosphere, Geophys. Res. Lett., 25, 2737-2740, 1998.

Regenauer-Lieb, K. and D. A. Yuen, Modeling shear zones in geological and planetary sciences: solid- and fluid-thermal-mechanical approaches, Earth Sci. Rev., 63, 295-349, 2003.

Regenauer-Lieb, K. and D. A. Yuen, Positive feedback of interacting ductile faults from coupling of equation of state, rheology and thermalmechanics, Phys. Earth Planet. Inter., 142, 113-135, 2004.

Ross, M. N. and G. Schubert, Tidal heating in an internal heating model of Europa, Nature, 325, 133-144, 1987.

Schiesser, W. E., Computational Mathematics in Engineering and Applied Science: ODEs, DAEs, and PDEs, Chapter 4, CRC, Florida, 1994.

Segatz, M., T. Spohn, M. N. Ross, and G. Schubert, Tidal dissipation, surface heat flow, and figure of viscoelastic models of Io, Icarus, $\mathbf{7 5}$, 187-206, 1988.

Siddall, M., E. J. Rohling, A. Almogi-Labin, Ch. Hemleben, D. Meischner, I. Schmelzer, and D. A. Smeed, Sea-level fluctuations during the last glacial cycle, Nature, 423, 813-814, 2003.

Stein, C. A., Heat flow of the Earth, in Global Earth Physics: A Handbook of Physical Constants, edited by T. J. Ahrens, pp. 144-158, AGU, 1995. van den Berg, A. P. and D. A. Yuen, The role of shear heating in lubricating mantle flow, Earth Planet. Sci. Lett., 151, 33-42, 1997.

Wu, P. and W. R. Peltier, Viscous gravitational relaxation, Geophys. J. R. Astr. Soc., 70, 435-485, 1982.

Yuan, D., H. Cheng, R. L. Edwards, C. A. Dykoski, M. J. Kelly, M. Zhang, J. Qing, Y. Lin, W. Wang, J. Wu, J. A. Dorale, Z. An, and Y. Cai, Timing, duration and transitions of the last interglacial Asian monsoon, Science, 304, 575-578, 2004.

L. Hanyk (e-mail: ladislav.hanyk@mff.cuni.cz), C. Matyska, and D. A. L.
Yuen 